\title{
Fully-Developed Circular-Pipe Flow of a Non-Newtonian Pseudoplastic Fluid
}

\author{
Lun-Shin Yao $^{1}$, Md. Mamun Molla ${ }^{2, *}$, S. Ghosh Moulic ${ }^{3}$ \\ ${ }^{1}$ School for Engineering of Matter, Transport and Energy, Arizona State University, Arizona, 85287, USA \\ ${ }^{2}$ Department of Electrical Engineering \& Computer Science, North South University, Dhaka-1229, Bangladesh \\ ${ }^{3}$ Department of Mechanical Engineering, Indian Institute of Technology, Kharagpur, Kharagpur 721302, India \\ *Corresponding Author: mmamun@northsouth.edu, mmamun@gmail.com
}

Copyright (C2013 Horizon Research Publishing All rights reserved.

\begin{abstract}
A fully-developed flow of a non-Newtonian pseudoplastic fluid through a circular pipe has been studied using a four-parameter model, as an example, for the shear-rate dependent apparent viscosity. The model used in this investigation is a modification of the two-parameter Ostwald-de Waele power law [1], which correctly represents the lower and upper regions of Newtonian behavior characteristic of pseudoplastic polymer melts and solutions. Since there has only one set complete experimental data available for the shear-rate dependent viscosity, we use them to show that a perfect match can be achieved between the modified power-law viscosity model and the experimental data. Such a perfect match is required for an accurate prediction of the flow behavior in internal flow problems.
\end{abstract}

Keywords Fully developed pipe flow, nonNewtonian fluid, modified power-law visclsoity model

\section{Introduction}

A non-dimensional parameter governing the flow has been identified, which is the ratio of the length scale, introduced by the empirical correlation of the variable viscosity and the radius of the pipe. This length scale exists in order to properly model the physics associated with variable viscosity. Its necessity was first described in several recent publications for boundary-layer flows. The current paper is, however, the first analysis to show the existence of this length scale also for internal flow problems. It is our opinion that this kind of length scales must exist for all problems whose formulation would be incomplete without empirical correlations to model the physics of problems, but have rarely been discussed. The results indicate that there are three regimes of flow. When the value of the non-dimensional parameter is small, the fluid behaves like a Newtonian fluid with a viscosity equal to the zero-shear-rate viscosity, as the shear-rate magnitude is not large enough to induce non-Newtonian behavior. At very high values of the non-dimensional parameter, the fluid behaves like a Newtonian fluid with a viscosity equal to the zero-shearrate viscosity in the central region of the pipe, and like a Newtonian fluid with a viscosity equal to the infiniteshear-rate viscosity in the outer region of the pipe adjacent to the pipe wall; power-law behavior is seen in the middle region of the pipe, between the central and outer regions. At intermediate values of the non-dimensional parameter, there are two distinct regions of flow: an inner central region where the fluid behaves like a Newtonian fluid with a viscosity equal to the zero-shear-rate viscosity, and an outer region adjacent to the pipe wall where the fluid behaves like a power-law fluid. The results have been compared with those predicted by the Ostwald-de Waele power-law model [1]; the superiority and necessity of the modified model is clearly demonstrated.

Non-Newtonian fluids may be broadly classified into three categories: viscous time-independent fluids, viscous time-dependent fluids and viscoelastic fluids. Fluids for which the shear stress at a point is determined only by the value of the instantaneous shear rate at that point are known as 'purely viscous' or 'timeindependent' fluids. These fluids are also referred to as 'inelastic' fluids or 'generalized Newtonian fluids'. Fluids for which the shear stress at a point depends on the instantaneous shear rate at that point as well as the duration of shearing and kinematic history are called viscous 'time-dependent' non-Newtonian fluids. Fluids that exhibit the characteristics of both fluids and elastic solids are referred to as 'viscoelastic' fluids. A comprehensive review of non-Newtonian fluid dynamics may be found in the lecture notes of [2]. In this investigation, purely viscous, time-independent or generalized Newtonian fluids have been considered.

The generalized Newtonian fluid model is obtained by replacing the viscosity of the Newtonian fluid by an apparent or non-Newtonian viscosity that depends on the magnitude of the rate-of-strain tensor, in Stokes law of viscosity and the Navier-Stokes equations [3]. If the apparent viscosity of a generalized Newtonian fluid decreases with an increase in the magnitude of the shear rate, the fluid is referred to as a shear-thinning or pseu- 
doplastic fluid. If the apparent viscosity of a generalized Newtonian fluid increases with an increase in the magnitude of the shear rate, the fluid is referred to as a shear-thickening or dilatant fluid.

The simplest constitutive equation relating the apparent viscosity of a generalized Newtonian fluid to the shear rate is the two-parameter Ostwald-de Waele power-law [1] [4] [5] [6]. The limitations of the power-law model are well known. For shear-thinning or pseudoplastic fluids, the power law yields a physically unrealistic prediction of infinite apparent viscosity in the limiting case of zero shear rates and a zero apparent viscosity in the limit of infinite shear rate. The nonNewtonian viscosity of shear-thickening or dilatant fluids predicted by the power-law model is zero in the limit of zero shear-rate and becomes unbounded in the limiting case of infinite shear rate. Infinite shear rates occur at the leading edge of forced convection boundarylayer flows. Zero shear rates occur near the outer edge of boundary-layer flows, at the leading edge of natural convection boundary-layer flows, and at the centerline of axisymmetric pipe flows. The Ostwald-de Waele power-law model introduces non-physical singularities in boundary-layer formulations, at the leading edge and near the outer edge of the boundary-layer. Some authors assumed that this is a non-removal singularity and they starting the integration slightly downstream from the leading edge. For example, Huang etal. [7] investigated the boundary-layer heat transfer problem with powerlaw fluids. Since a sigular point exists at the leading egde $(\xi=0)$, they starting their integration from $\xi=10^{-10}$ and produced the unrealisting boundary-layer solutions. However, in spite of its limitations, the power-law model is widely used in engineering applications, because of its simplicity.

Most of the non-Newtonian fluids used in engineering applications are pseudoplastic or shear-thinning. Familiar examples include printing inks, paints, polymer melts and solutions. Experimental data indicates that a typical pseudoplastic fluid behaves as a Newtonian fluid at low shear rates and at very high shear rates [1] [4] [5] [6] [8]. The apparent viscosity is constant at low shearrates; this constant value of the apparent viscosity is referred to as the zero-shear-rate viscosity. As the shear rate is increased beyond a certain value, the apparent viscosity begins to decrease. At very high shear-rates, the fluid again behaves like a Newtonian fluid with a constant apparent viscosity referred to as the infiniteshear-rate viscosity.

A modified power-law viscosity model was proposed by [9], in which the Ostwald-de Waele power law is used to determine the non-Newtonian viscosity for a certain range of the magnitude of the shear rate; outside this range of shear rate magnitude, the viscosity was taken to be constant. When the magnitude of the shear rate is lower than a certain threshold shear-rate, referred to as the lower threshold shear rate, the viscosity is set equal to the zero-shear-rate viscosity. When the magnitude of the shear rate exceeds a certain threshold shear-rate, referred to as the upper threshold shear rate, the viscosity is set equal to the infinite-shear-rate viscosity. The modified power-law does not predict zero or infinite values of the apparent viscosity in the limiting cases of zero and infinite shear rates. Thus, the singularities introduced into the boundary-layer formulation by the popular Ostwald-de Waele power-law model do not appear when the modified power-law is used [9] [10] [11] [12] [13] [14] [15] [16]. The studies of external boundary-layer flows of non-Newtonian fluids [9] [10] [11] [12] [13] [14] [15] [16] also established that self-similar solutions of the boundary-layer equations do not exist, as the power law introduces a characteristic length scale in the problem formulation.

In this investigation, the modified power-law proposed by [9] has been used to study fully-developed flow in a circular pipe, which is a typical example of internal flow. It has been shown that the characteristic length scale introduced by the popular Ostwald-de Waele power-law model appears in the formulation of internal flow problems as well. This introduces a new non-dimensional parameter in the problem. The study of flow of nonNewtonian fluids in pipes is of fundamental importance, and has applications in chemical and process industries. Results have been obtained for a shear-thinning polymer solution for which experimental data for the apparent viscosity is available over a wide range of shear rates [8]. The empirical parameters of the modified powerlaw have been determined to fit the data for the apparent viscosity reported by [8], which, to our knowledge, is the only set of complete data available in the open literature. The results have been compared with those predicted using the power-law model.

\section{Formulation of the problem}

The fully-developed flow of a constant-density nonNewtonian fluid through a circular pipe of radius ' $a$ ' has been studied. It is convenient to use cylindrical coordinates $(\bar{r}, \bar{z})$ to describe the axisymmetric flow, where $\bar{z}$ is a coordinate along the axis of the pipe, and $\bar{r}$ is the radial coordinate. The flow is considered to be steady, and laminar. Thus, the swirl component of velocity is zero, and the axial component of velocity, $\bar{w}$, is a sole function of the radial coordinate $\bar{r}$. The no-penetration boundary condition at the surface of the pipe and the continuity equation imply that the radial component of velocity is zero in the fully developed region of the flow.

The generalized Newtonian fluid model has been used in this investigation. Thus, the equations describing balance of momentum are the Navier-Stokes equations with a shear-rate-dependent viscosity. The balance of momentum in the radial and azimuthal directions show that the modified pressure, $\bar{P}=\bar{p}-\rho \mathbf{g} \cdot \mathbf{r}$, is independent of $\bar{r}$, and hence, is a sole function of $\bar{z}$. Here, $\bar{p}$ is the pressure, $\rho$ is the fluid density, $\mathbf{g}$ is the gravitational acceleration and $\mathbf{r}$ is the position vector. The balance of momentum in the axial direction reduces to (see [3])

$$
\frac{1}{\bar{r}} \frac{d}{d \bar{r}}\left(\mu \bar{r} \frac{d \bar{w}}{d \bar{r}}\right)=-\frac{d \bar{P}}{d \bar{z}},
$$

where $\mu$ is the non-Newtonian viscosity. A separation of variables argument indicates that the axial gradient of the modified pressure, $\frac{d \bar{P}}{d \bar{z}}$, is a constant. Equation 
(1) has to be solved subject to the no-slip boundary condition

$$
\bar{w}(a)=0,
$$

at the surface, $\bar{r}=a$, of the pipe. Rotational symmetry of the flow requires that the velocity distribution, $\bar{w}(\bar{r})$, satisfy the condition

$$
\frac{d \bar{w}}{d \bar{r}}(0)=0,
$$

at the axis, $\bar{r}=0$, of the pipe. The condition (3) may be viewed as a regularity condition at the singular point, $\bar{r}=0$, of the differential equation (1). The apparent viscosity, $\mu$, of the generalized Newtonian fluid depends on the magnitude, $|\bar{\gamma}|$, of the shear-rate

$$
\bar{\gamma}=\frac{d \bar{w}}{d \bar{r}}
$$

Thus, equation (1) is a nonlinear ordinary differential equation. In this investigation, the constitutive relation proposed by [9], which is a modification of the power-law model, has been used to relate the apparent viscosity to the magnitude of the shear-rate.

\section{A. The Power-Law (PL) Viscosity Model}

The shear-rate dependent viscosity for a power-law fluid is given by [1]

$$
\mu=\bar{f}_{P L}(|\bar{\gamma}|)
$$

where

$$
\bar{f}_{P L}(|\bar{\gamma}|)=C|\bar{\gamma}|^{n-1} .
$$

Equation (6) involves two parameters that characterize the fluid: a dimensional parameter, $C$, and a dimensionless parameter, $n$. The dimensions of the parameter, $C$, referred to as the fluid consistency, depends on the power-law index, $n$. The apparent viscosity predicted by the Ostwald-de Waele power-law model (6) is independent of the shear-rate when $n=1$. This corresponds to the case of a Newtonian fluid. Equation (6) shows that the fluid consistency, $C$, for the special case of a Newtonian fluid $(n=1)$ is equal to the viscosity. When $n>1$, the fluid is said to be dilatant or shear-thickening. Equation (6) predicts that the apparent viscosity of dilatant fluids goes to zero as the shear-rate approaches zero, and increases without bound as the shear-rate is increased. When $n<1$, the fluid is said to be pseudoplastic or shear-thinning. Equation (6) indicates that the apparent viscosity of pseudoplastic fluids is infinite in the limit of zero shear- rate, and approaches zero as the shear-rate is increased.

Experimental data indicates that many nonNewtonian fluids, such as polymeric fluids, exhibit Newtonian behavior at very low and very high shear rates, with constant apparent viscosities referred to as zero-shear-rate viscosity and infinite-shear-rate viscosity respectively [1] [4] [5] [6] Boger1977; the power-law variation of the viscosity, described by equation (6), occurs only in an intermediate range of shear-rates. In spite of the unrealistic prediction of zero and infinite viscosities, the Ostwald-de Waele power-law model is widely used in engineering work, as many problems can be solved analytically when the variation of the viscosity with shear-rate is described by equation (6).

\section{B. The Modified Power-Law (MPL) Viscosity Model}

To remove the unrealistic prediction of zero and infinite viscosity, [9] proposed a simple modification of the Ostwald-de Waele power law. The variation of the apparent viscosity with shear-rate in the modified powerlaw proposed by [9] is described by the constitutive equation

$$
\mu=\bar{f}_{M P L}(|\bar{\gamma}|),
$$

where

$$
\bar{f}_{M P L}(|\bar{\gamma}|)= \begin{cases}\mu_{0}, & |\bar{\gamma}| \leq \bar{\gamma}_{1} \\ C|\bar{\gamma}|^{n-1}, & \bar{\gamma}_{1} \leq|\bar{\gamma}| \leq \bar{\gamma}_{2} \\ \mu_{\infty}, & |\bar{\gamma}| \geq \bar{\gamma}_{2}\end{cases}
$$

where $\bar{\gamma}_{1}$ and $\bar{\gamma}_{2}$ are two threshold shear-rates, $\mu_{0}$ is the zero-shear-rate viscosity and $\mu_{\infty}$ is the infiniteshear-rate viscosity. The Ostwald-de Waele power-law is used to determine the viscosity when the shear-rate magnitude falls in the range $\bar{\gamma}_{1} \leq|\bar{\gamma}| \leq \bar{\gamma}_{2}$. The viscosity is assumed to be independent of the shear-rate when the magnitude of the shear-rate is outside the range $\bar{\gamma}_{1} \leq|\bar{\gamma}| \leq \bar{\gamma}_{2}$. When the magnitude of the shear rate is smaller than the lower threshold shear-rate, $\bar{\gamma}_{1}$, the viscosity predicted by equation (8) is the zero-shear-rate viscosity. The viscosity is set equal to the infinite-shear-rate viscosity when the magnitude of the shear rate is larger than the upper threshold shear-rate, $\bar{\gamma}_{2}$.

The modified power-law correlation, given by equation (8), involves six parameters characterizing the fluid: $C, n, \bar{\gamma}_{1}, \bar{\gamma}_{2}, \mu_{0}$ and $\mu_{\infty}$. It is worth noting that when $\bar{\gamma}_{1}=0$ and $\bar{\gamma}_{2}$ is infinite, the power law covers the entire range of shear-rates. Thus, the Ostwald-de Waele power-law may be viewed as a special case of equation (8) when $\bar{\gamma}_{1}=0$ and $\bar{\gamma}_{2}$ is infinite. For this special case, when $\bar{\gamma}_{1}=0$ and $\bar{\gamma}_{2}$ is infinite, the values of $\mu_{0}$ and $\mu_{\infty}$ are not required for determining the non-Newtonian viscosity.

The function, $\bar{f}_{M P L}(|\bar{\gamma}|)$, defined by equation (8), is assumed to be a continuous function of $|\bar{\gamma}|$. When $\bar{\gamma}_{1}$ is non-zero, the function, $\bar{f}_{M P L}(|\bar{\gamma}|)$, is continuous at $|\bar{\gamma}|=$ $\bar{\gamma}_{1}$ if the following relation is satisfied:

$$
\bar{\gamma}_{1}=\left[\frac{\mu_{0}}{C}\right]^{\frac{1}{n-1}} .
$$

When $\bar{\gamma}_{2}$ is finite, the function, $\bar{f}_{M P L}(|\bar{\gamma}|)$, is continuous at $|\bar{\gamma}|=\bar{\gamma}_{2}$ if

$$
\bar{\gamma}_{2}=\left[\frac{\mu_{\infty}}{C}\right]^{\frac{1}{n-1}} .
$$

Equation (9) gives a relation between $\bar{\gamma}_{1}$ and $\mu_{0}$ when $\bar{\gamma}_{1}$ is non-zero, while equation (10) gives a relation between $\bar{\gamma}_{2}$ and $\mu_{\infty}$ when $\bar{\gamma}_{2}$ is finite. Thus, the modified powerlaw correlation involves four independent parameters. When $\bar{\gamma}_{1}$ is non-zero and $\bar{\gamma}_{2}$ is finite, the parameters $\left(C, n, \mu_{0}\right.$ and $\left.\mu_{\infty}\right)$ may be used as the independent set of parameters in the modified power-law, with $\bar{\gamma}_{1}$ and 
$\bar{\gamma}_{2}$ determined from equations (9) and (10).

The modified power-law correlation (8) may be expressed as

$$
\mu=\mu_{0} f_{M P L}(|\Gamma|),
$$

where $\Gamma$ is a non-dimensional shear-rate defined by

$$
\Gamma=\bar{\gamma} t_{S}
$$

$t_{S}$ is a characteristic time-scale defined by

$$
t_{S}=\left(\frac{C}{\mu_{0}}\right)^{\frac{1}{n-1}}
$$

$f_{M P L}(|\Gamma|)$ is a non-dimensional function defined by

$$
f_{M P L}(|\Gamma|)= \begin{cases}1, & |\Gamma| \leq \Gamma_{1} \\ |\Gamma|^{n-1}, & \Gamma_{1} \leq|\Gamma| \leq \Gamma_{2} \\ \mu_{R}, & |\Gamma| \geq \Gamma_{2}\end{cases}
$$

and $\mu_{R}$ is the viscosity ratio

$$
\mu_{R}=\frac{\mu_{\infty}}{\mu_{0}} .
$$

The non-dimensional model constants, $\Gamma_{1}$ and $\Gamma_{2}$, are respectively the lower and upper non-dimensional threshold shear rates, defined by

$$
\Gamma_{1}=\bar{\gamma}_{1} t_{S}
$$

and

$$
\Gamma_{2}=\bar{\gamma}_{2} t_{S}
$$

The time scale, $t_{S}$, is a property of the fluid that depends on $C, \mu_{0}$ and $n$. Equations (9) and (13) imply that

$$
t_{S}=\frac{1}{\bar{\gamma}_{1}}
$$

Thus, the characteristic time scale, $t_{S}$, is the reciprocal of the lower threshold shear-rate. It follows from equation (16), (17) and (18) that the lower non-dimensional threshold shear-rate is

$$
\Gamma_{1}=1
$$

and the upper non-dimensional threshold shear-rate is

$$
\Gamma_{2}=S,
$$

where

$$
S=\frac{\bar{\gamma}_{2}}{\bar{\gamma}_{1}}
$$

The ratio of the upper and lower threshold shear-rates, $S$, is a property of the fluid, and may be expressed in terms of $\mu_{0}, \mu_{\infty}$ and $n$ as

$$
S=\left[\frac{\mu_{\infty}}{\mu_{0}}\right]^{\frac{1}{n-1}}
$$

The non-dimensional modified power-law function, $f_{M P L}(|\Gamma|)$, thus involves two non-dimensional parameters characterizing the fluid: $n$ and $\frac{\mu_{\infty}}{\mu_{0}}$, or equivalently, $n$ and $S$.

\section{Non-dimensional equations and boundary conditions}

The axial momentum equation may be nondimensionalized using the radius, $a$, of the pipe as the length scale, the zero-shear-rate viscosity, $\mu_{0}$, as the reference viscosity and a velocity scale, $\bar{W}_{0}$, defined by

$$
\bar{W}_{0}=-\frac{a^{2}}{4 \mu_{0}} \frac{d \bar{P}}{d \bar{z}} .
$$

Introducing a non-dimensional shear-rate dependent viscosity defined by

$$
D=\frac{\mu}{\mu_{0}}
$$

a non-dimensional axial velocity defined by

$$
w=\frac{\bar{w}}{\bar{W}_{0}},
$$

and a non-dimensional radial coordinate defined by

$$
r=\frac{\bar{r}}{a}
$$

the axial momentum equation (1) may be written in non-dimensional form as

$$
\frac{d}{d r}\left(r D \frac{d w}{d r}\right)=-4 r
$$

The associated no-slip boundary condition may be expressed in non-dimensional form as

$$
w(1)=0
$$

at the surface, $r=1$, of the pipe. The regularity condition (3) may be written in non-dimensional form as

$$
\frac{d w}{d r}(0)=0
$$

at the axis, $r=0$, of the pipe. The shear-rate, required for determination of the non-Newtonian viscosity, may be written as

$$
\bar{\gamma}=\frac{\bar{W}_{0}}{a} \gamma
$$

where

$$
\gamma=\frac{d w}{d r}
$$

The velocity scale defined by equation (23) depends on the constant applied pressure gradient, the radius of the pipe and the reference viscosity. For the special case of a Newtonian fluid $(n=1)$, the reference velocity, $\bar{W}_{0}$, defined by equation $(23)$, is the velocity at the centre-line of the pipe. Since the shear-rate is zero at the centerline of the pipe, as indicated by equation (3), the viscosity at the centerline is the zero-shear-rate viscosity. Thus, the reference viscosity used in the non-dimensionalization is the viscosity at the centerline.

The velocity distribution, $w(r)$, may be determined by solving equation (27) subject to the no-slip boundary condition (28) and the regularity condition (29). To complete the problem formulation, one needs to specify the constitutive equations relating the non-dimensional viscosity, $D$, to the non-dimensional shear rate, $\gamma$, given 
by equation (31). For the modified power-law viscosity model, the non-dimensional viscosity, $D$, is given by

$$
D=f_{M P L}(|\Gamma|)
$$

where $f_{M P L}(|\Gamma|)$ is the function defined by equation (14). The non-dimensional shear-rate, $\Gamma$, appearing in equation (32), is related to the non-dimensional shearrate, $\gamma$, introduced by the velocity scale, $\bar{W}_{0}$, and the length scale $a$, by

$$
\Gamma=A \gamma
$$

where $A$ is a non-dimensional parameter defined by

$$
A=\frac{\bar{W}_{0} t_{S}}{a} .
$$

This indicates that the usual non-dimensionalization used in the study of fully-developed flow of a Newtonian fluid through a circular pipe cannot describe the flow of a non-Newtonian fluid. An additional parameter, $A$, is required to fully characterize the flow of non-Newtonian pseudoplastic fluids.

The non-dimensional parameter, $A$, may be expressed as the ratio of two length scales:

$$
A=\frac{L}{a}
$$

where $L$ is a characteristic length scale defined by

$$
L=\bar{W}_{0} t_{S}
$$

Using equations (18) and (34), the parameter $A$ may also be written as $A=\left(\bar{W}_{0} / a\right) / \bar{\gamma}_{1}$. Since $\left(\bar{W}_{0} / a\right)$ is a measure of the magnitude of the shear-rate, $\frac{d \bar{w}}{d \bar{r}}$, the parameter $A$ may be interpreted as the ratio of the shear-rate magnitude to the lower threshold shear-rate, $\bar{\gamma}_{1}$. Thus, $A$ may be viewed as a non-dimensional shear-rate parameter.

For the power-law viscosity model, the nondimensional viscosity is given by

$$
D=f_{P L}(|\Gamma|)
$$

where

$$
f_{P L}(|\Gamma|)=|\Gamma|^{n-1}
$$

The solution of the momentum equation for the powerlaw viscosity model is given in standard texts, e.g. Bird2002. The velocity distribution for the power-law viscosity model may be expressed in non-dimensional form as

$$
w(r)=\frac{n}{n+1}\left(\frac{2}{A^{n-1}}\right)^{\frac{1}{n}}\left(1-r^{\frac{n+1}{n}}\right) .
$$

The velocity distribution for the modified-power law viscosity model has been determined by a numerical method, as described in section 3 .

\section{$3 \quad$ Numerical Method}

Integration of equation (27) and application of the zero shear-rate condition (29) at the axis of the pipe results in

$$
\frac{d w}{d r}=-\frac{2 r}{D}
$$

Equation (40) was integrated numerically, using the trapezoidal rule. This leads to

$$
w_{j}=w_{j+1}+\left(\left[\frac{r}{D}\right]_{j+1}+\left[\frac{r}{D}\right]_{j}\right) \Delta r,
$$

where $w_{j}$ is the approximate value of $w\left(r_{j}\right)$,

$$
\begin{gathered}
r_{j}=(j-1) \Delta r, j=1,2, \ldots, N, \\
\Delta r=\frac{1}{N-1},
\end{gathered}
$$

and $N$ is the number of grid points. The no-slip boundary condition implies that

$$
w_{N}=0
$$

Equation $((41))$ is solved iteratively with $D$ calculated using equation (32), sequentially for $j=N-1$, $N-2, \ldots, 1$. The discrete boundary condition (44) is enforced in equation (41) for $j=N-1$. The iterations were continued until the difference between successive iterates was less than a tolerance, which has taken to be $10^{-6}$. The value of $\Delta r$ was taken to be 0.001 .

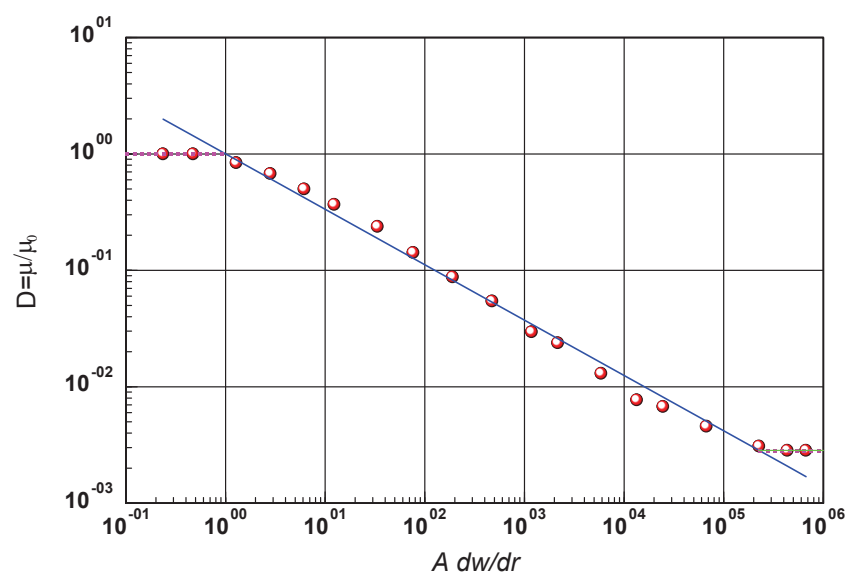

Figure 1. Variation of the non-dimensional viscosity, $D=\frac{\mu}{\mu_{0}}$, with the magnitude of the non-dimensional shearrate, $|\Gamma|=|\mathrm{Adw} / \mathrm{dr}|$. The circles represent experimental data from [8]. The reference viscosity is $\mu_{0}=1.42 \mathrm{kgm}^{-1} \mathrm{~s}^{-1}$.

\section{Results and discussion}

Results are presented for a shear-thinning polymer solution for which data for the variation of the apparent viscosity, measured over a large range of shear-rates, has been reported by Boger1977. The zero-shear-rate viscosity and the infinite-shear-rate viscosity of the $0.4 \%$ polyacrylamide solution used in the experiments of [8] are $1.42 \mathrm{~Pa} \mathrm{~s}$ and $4 \times 10^{-3} \mathrm{~Pa}$ s respectively. Thus, the ratio of the infinite-shear-rate viscosity to the zero-shearrate viscosity for this polymer solution is $\mu_{R}=2.82 \mathrm{x}$ 
$10^{-3}$. Data for the variation of the apparent viscosity with the shear rate, taken from Boger's experimet [8], has been plotted in non-dimensional form in Figure 1 which shows the variation of the non-dimensional viscosity, $D$, with the non-dimensional shear-rate magnitude, $|\Gamma|$. The variation of $\mathrm{D}$ with $|\Gamma|$, predicted by the nondimensional modified power law function, $f_{M P L}(|\Gamma|)$, defined by equation (14), is also plotted in Figure 1, for $\mu_{R}$ $=2.82 \times 10^{-3}$ and $n=0.5242$. A glance at Figure 1 reveals that the modified power law, $D=f_{M P L}(|\Gamma|)$, with $n=0.5242$ and $\mu_{R}=2.82 \times 10^{-3}$, is an excellent fit to the experimental data points indicated by the circles. The ratio of the upper threshold shear rate to the lower threshold shear rate is $S=2.29 \times 10^{5}$, when $n=0.5242$ and $\mu_{R}=2.82 \times 10^{-3}$.

Results have been obtained for several values of the non-dimensional flow parameter, $A$. The radial distribution of the axial velocity, non-dimensional shear-rate magnitude and non-dimensional viscosity are shown in Figures 2, 3 and 4, respectively. The results, predicted using the power-law are also shown in the figures for comparison with the results obtained using the modified power-law. The solid lines in these figures indicate the prediction using the modified power-law, while the dashed lines indicate the prediction of the power-law.
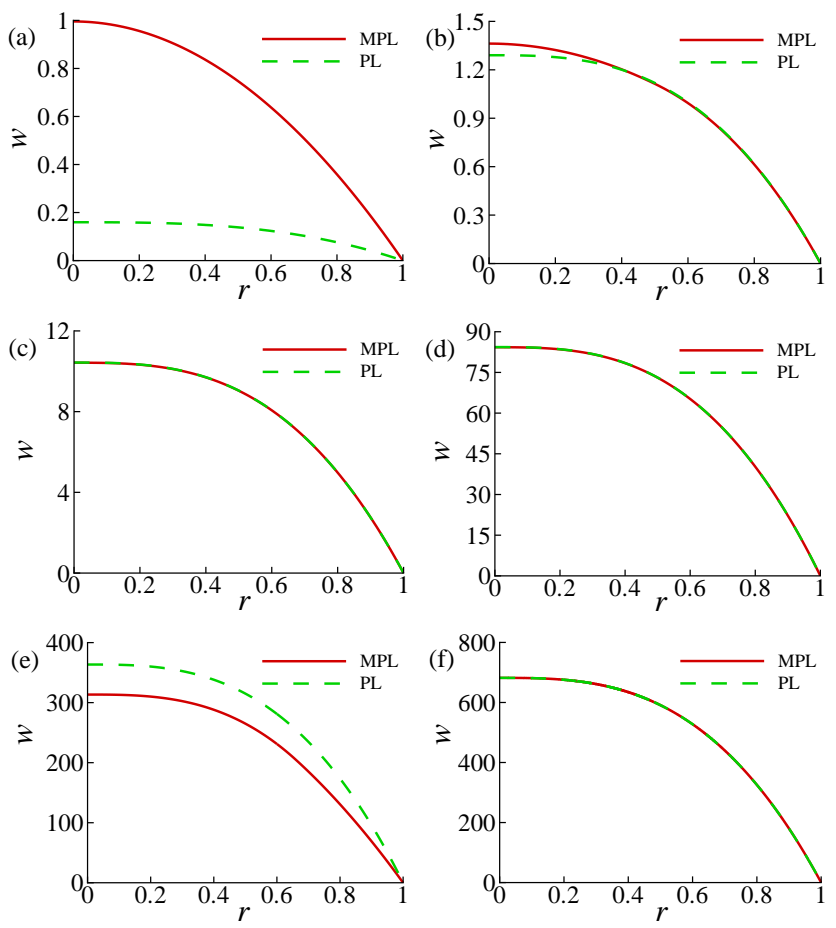

Figure 2. Axial velocity distribution, $w$, for (a) $A=0.1$ (b) $A=$ 1 (c) $A=10$ (d) $A=100$ (e) $A=500$ and (f) $A=1000$.

Figures 2(a)-(f) show the distribution of axial velocity, $w$, for $A=0.1,1,10,100,500$ and 1000 respectively. Figure 2(a) displays the radial variation of the axial velocity when $A=0.1$. A glance at Figure 2(a) reveals that the velocity predicted by the power law is much smaller than the velocity predicted by the modified power-law at all points in the flow field. Figure $2(\mathrm{~b})$ shows the radial variation of the axial velocity for $A=1$. The figure indicates that the velocity predicted by the power-law is slightly smaller than the velocity predicted by the modified power-law in the region close to the axis of the pipe. The velocity profiles predicted by the power-law and the modified power-law are almost identical in the outer region of the pipe when $A=1$. The distribution of the axial velocity for $A=10$ and $A=100$ are shown in Figures 2(c) and (d), respectively. The figures show that for these two cases the velocity profiles predicted by the power-law and modified power law-are identical, on the scale of the graphs, at all points in the flow field. When $A=500$, the velocity predicted by the power-law is everywhere higher than the velocity predicted by the modified power-law. This is indicated by Figure 2(e). A similar trend is displayed by Figure 2(f) which shows the velocity distribution for $A=1000$. The difference in the magnitude of the velocities predicted by the modified power-law and the power-law is larger when $A=1000$, compared to the difference in the two predictions when $A=500$.
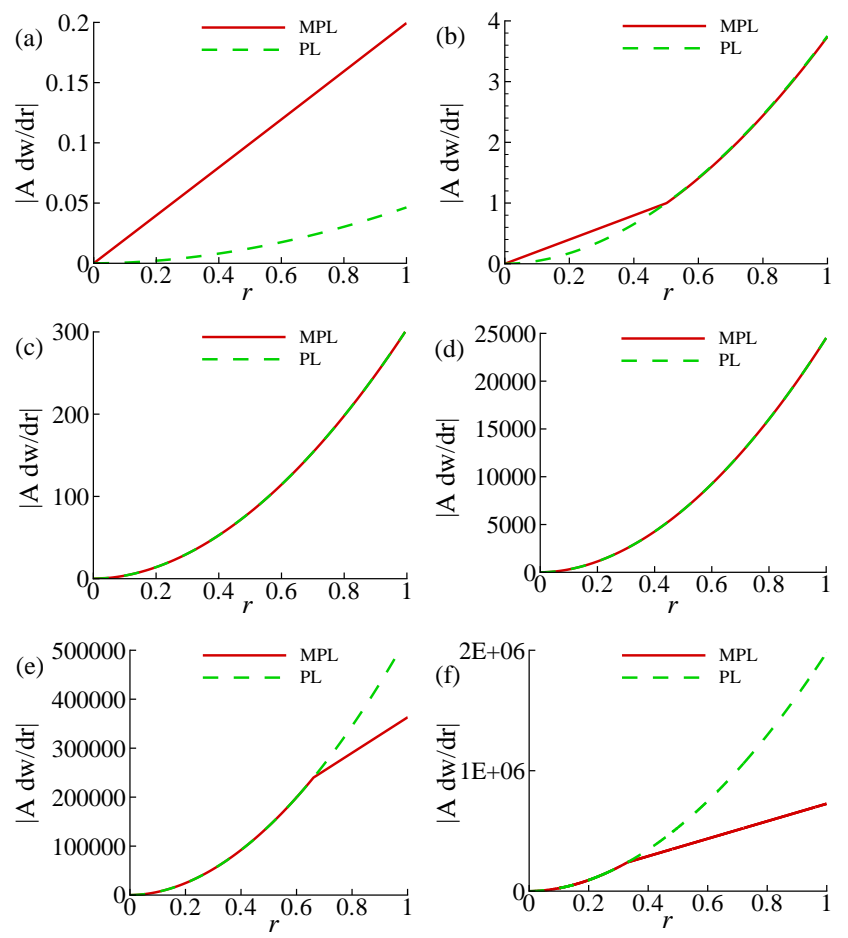

Figure 3. Radial variation of the shear-rate magnitude, $|\Gamma|=\mid$ Adw $/ \mathrm{dr} \mid$, for (a) $A=0.1$ (b) $A=1$ (c) $A=10$ (d) $A=$ 100 (e) $A=500$ and (f) $A=1000$.

In order to explain the nature of the graphs shown in Figures 2(a)-(f), the radial distributions of non-dimensional shear-rate magnitude, $|\Gamma|$, and nondimensional viscosity, $D$, are plotted in Figures 3 and 4 respectively. Figure 3(a) indicates that the radial distribution of the non-dimensional shear-rate magnitude predicted by the modified power-law is linear, and higher than the shear-rate magnitude predicted by the power-law at all points in the flow field. A glance at Figure 3(a) reveals that the non-dimensional shear-rate magnitude predicted by the modified power-law is everywhere less than the non-dimensional lower threshold shear-rate, $\Gamma_{1}=1$. Consequently, the value of the nondimensional viscosity predicted by the modified powerlaw is $D=1$ at all points, as indicated by Figure 4(a). The velocity profile predicted by the modified powerlaw is thus identical to that of a Newtonian fluid with a 
constant viscosity, $D=1$, flowing through the pipe. Figure 4(a) shows that the non-dimensional viscosity predicted by the power-law is higher than the constant nondimensional viscosity, $D=1$, predicted by the modified power-law at all points in the flow field. Due to the higher viscosity, the velocity predicted by the power-law is much smaller than the velocity predicted by the modified power-law.

Figure 3(b) indicates that the non-dimensional shearrate magnitude, predicted by the modified power-law, increases linearly from a zero value at the axis, $r=0$, to $|\Gamma|=\Gamma_{1}=1$ at $r=0.5$, when $A=1$. For $r<$ 0.5 , the shear-rate magnitude predicted by the modified power-law is higher than that predicted by the power-law. For $r>0.5$, the non-dimensional shear-rate magnitudes predicted by the modified power-law and the power-law are almost identical. The magnitude of the non-dimensional shear-rates predicted by both the laws is everywhere less than the non-dimensional upper threshold shear-rate, $\Gamma_{2}=S$. Figure 4(b) shows the radial distribution of the non-dimensional viscosity for $A=1$. The value of the non-dimensional viscosity predicted by the modified power-law is $D=1$ for $0 \leq r \leq 0.5$, as the non-dimensional shear-rate magnitude, $|\Gamma|$, in this region is less than the non-dimensional lower threshold shear-rate. The viscosity predicted by the modified power-law decreases with increase in $r$, in the outer region, $0.5 \leq r \leq 1$, of the pipe. In this outer region, the viscosity predicted by the modified powerlaw and the power-law are almost identical. This is due to the fact that the shear-rate magnitudes predicted by the two laws are almost identical in this region, and fall in the range $\Gamma_{1} \leq|\Gamma| \leq \Gamma_{2}$. In the central region, $0 \leq r \leq 0.5$, of the pipe, the viscosity predicted by the power-law is higher than the viscosity predicted by the modified power-law. Consequently, the fluid velocity predicted by the power-law is smaller than the velocity predicted by the modified power-law, near the axis, as shown by Figure 2(b).

The radial distribution of the non-dimensional shearrate magnitudes for $A=10$ and $A=100$ are indicated in Figure 3(c) and (d) respectively. For these two cases, the prediction of the modified power-law coincides with the prediction of the power-law, on the scale of the graphs. The magnitude of the non-dimensional shear-rates is everywhere less than the non-dimensional upper threshold shear-rate. The radial distribution of non-dimensional viscosity for $A=10$ and $A=100$ are indicated in Figure $4(\mathrm{c})$ and (d) respectively. The figures show that the non-dimensional viscosity predicted by the modified power-law is constant in the central region of the pipe, with a value $D=1$, as in the case when $A=1$; powerlaw behavior is seen in the outer region of the pipe. The size of the central region, where the viscosity is constant, decreases with increase in the value of $A$. The viscosity predicted by the two laws is identical in the outer region of the pipe.

The radial distribution of the non-dimensional shearrate magnitude for $A=500$ is indicated in Figure 3(e). The figure indicates that the shear-rate magnitudes predicted by the two laws are almost identical in the central region of the pipe; in this region, the shear-rate magnitudes increase from a zero value at the axis to the upper
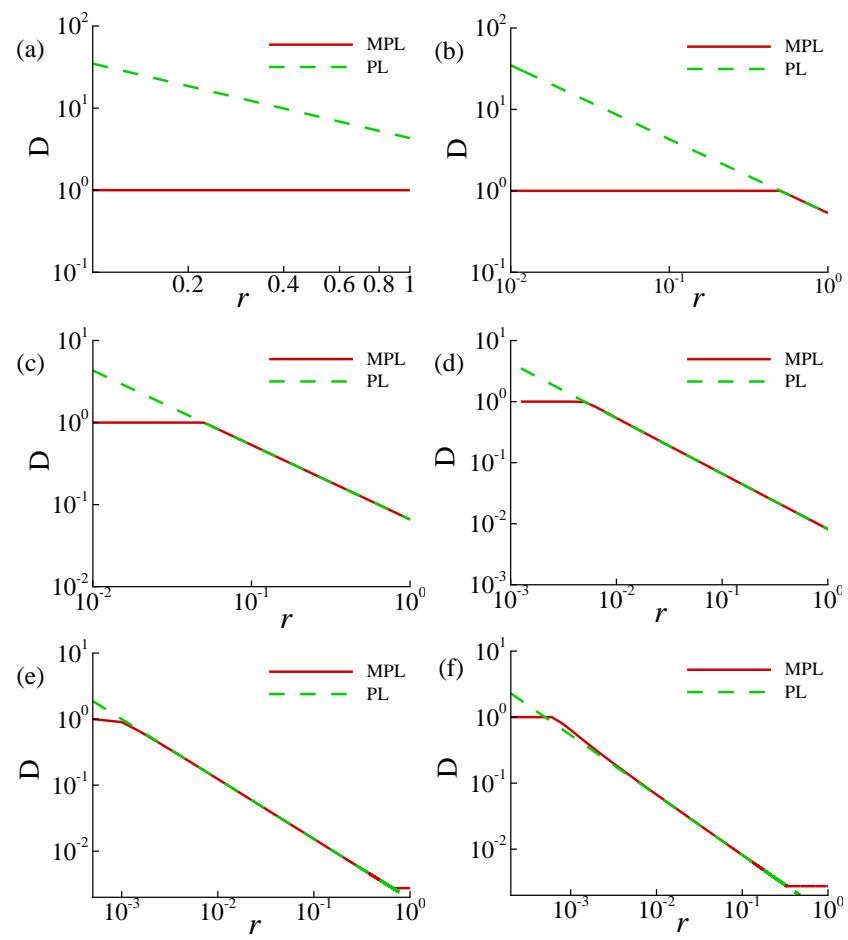

Figure 4. Viscosity distribution along the radius of pipe for (a) $A=0.1$ (b) $A=1$ (c) $A=10$ (d) $A=100$ (e) $A=500$ and (f) $A$ $=1000$.

threshold shear-rate at a certain radial location. Beyond this radial location, the shear-rate magnitude predicted by the modified power-law is lower than the shear-rate magnitude predicted by the power-law. In this outer region, the radial distribution of the shear-rate magnitude predicted by the modified power-law is linear. The radial distribution of the non-dimensional viscosity for $A=500$ is indicated in Figure 4(e). The figure shows that the viscosity predicted by the modified power-law is constant in the region of the pipe close to the axis, and in the region adjacent to the pipe wall. Power-law behavior is seen in the middle region of the pipe, between the region close to the axis and the region adjacent to the pipe wall. The value of the non-dimensional viscosity predicted by the modified power-law is $D=1$ in the region close to the axis, and $D=\mu_{R}$ in the region adjacent to the pipe wall. The non-dimensional viscosities predicted by the two laws are identical in the middle region. In the region close to the axis, the viscosity predicted by the power-law is higher than that predicted by the modified power-law. In the region adjacent to the pipe wall, the viscosity predicted by the modified powerlaw is higher than the viscosity predicted by the powerlaw. As a consequence, the magnitude of the velocity predicted by the modified power-law is smaller than the magnitude of the velocity predicted by the power-law.

Figure 3(f) shows the radial variation of the nondimensional shear-rate magnitude for $A=1000$. The corresponding distribution of the non-dimensional viscosity is plotted in Figure 4(f). Comparison of figures $3(\mathrm{f})$ and $3(\mathrm{e})$ and comparison of figures $4(\mathrm{f})$ and $4(\mathrm{e})$ indicate that the qualitative nature of the variation of $|\Gamma|$ and $D$ for $A=500$ and $A=1000$ are similar.

The non-dimensional average velocity through the pipe, defined as the non-dimensional volume flow rate 
per unit cross-sectional area of the pipe, was determined using

$$
w_{a v}=\frac{1}{\pi} \int_{0}^{1} 2 \pi r w(r) d r .
$$

The non-dimensional average velocity, $\left(w_{a v}\right)_{M P L}$, predicted by the modified power-law, and the nondimensional average velocity, $\left(w_{a v}\right)_{P L}$, predicted by the power-law, have been tabulated in Table 1 for several values of $A$. The table shows that the average velocity predicted the power-law is lower than the average velocity predicted by the modified power-law when $A$ is small. This is due to the fact that the viscosity predicted by the power-law is higher than the viscosity predicted by the modified power-law for small values of $A$. It is worth noting that the average velocity predicted by the modified power-law, for $A=0.1$, is the same as the average velocity for a Newtonian fluid. For $A=10$ and $A=100$, the average velocities predicted by the two laws are almost identical, since the velocity profiles are almost identical, as indicated by Figures 2(c) and (d). At large values of $A$, the average velocity predicted by the power-law is higher than the average velocity predicted by the modified power-law.

Table 1. Variation of the non-dimensional average velocities predicted by the power-law and the modified power-law for different A.

\begin{tabular}{|l|l|l|}
\hline$A$ & $\left(w_{a v}\right)_{P L}$ & $\left(w_{a v}\right)_{M P L}$ \\
\hline 0.1 & 0.09456 & 0.5 \\
1 & 0.7645 & 0.7703 \\
10 & 6.1810 & 6.1810 \\
100 & 49.97213 & 49.97214 \\
500 & 215.3580 & 171.8136 \\
1000 & 404.0141 & 177.1446 \\
\hline & \multicolumn{2}{|c}{} \\
\hline
\end{tabular}

\section{Conclusions}

The velocity distribution in fully-developed flow of a non-Newtonian pseudoplastic fluid through a circular pipe has been computed using a modified power-law. The parameters in the modified power-law have been determined to fit available experimental data for the variation of the non-Newtonian viscosity of a $0.4 \%$ polyacrylamide solution with shear-rate. A non-dimensional shear-rate parameter governing the flow has been determined; this non-dimensional parameter, $A$, depends on the applied pressure gradient. The results indicate that at low values of the shear-rate parameter, the velocity predicted by the modified power-law is higher than the velocity predicted by the power-law. At high values of the shear-rate parameter, the velocity predicted by the modified power-law is lower than the velocity predicted by the power-law. The velocities predicted by the modified power-law and the power-law are almost identical at intermediate values of the shear-rate parameter. At very low values of the shear-rate parameter, the velocity profile predicted by the modified power-law is identical to that for fully-developed flow of a Newtonian fluid with a constant viscosity equal to the zero-shear-rate viscosity. The importance of the shear-rate parameter, $A$, has been clearly demonstrated by the numerical results presented in the paper. One needs to carefully check the range of values of the parameter, $A$, before using the popular power-law model.

\section{REFERENCES}

[1] R. B. Bird, R. C. Armstrong, O. Hassager. Dynamics of Polymeric Liquids, Vol. 1, Fluid Mechanics, Wiley, New York, 1987, 2nd edition.

[2] J. Hinch. Non-Newtonian Geophysical Fluid Dynamics,2003 Program in Geophysical Fluid Dynamics, Woods Hole, MA 02543, USA, 2003.

[3] H. Hchlichting. Boundary-layer theory, McGraw-Hill, New York, USA, 1968.

[4] R. P. Chhabra, J. F. Richardson. Non-Newtonian Flow in the Process Industries, Fundamentals and Engineering Applications, Butterworth Heinemann, Oxford, 1999.

[5] R. P. Chhabra, J. F. Richardson. Non-Newtonian Flow and Applied Rheology: Engineering Applications, Butterworth Heinemann, Oxford, 2008, 2nd Edition.

[6] R. B. Bird, W. E. Stewart, W. E. Lightfoot. Transport Phenomena, Wiley, New York, 2002, 2nd Edition.

[7] J-M. Huang, J-S. Huang, Y-L. Chon, C-K. Chen. Effects of Prandtl number on Free convection Heat Transfer from a Vertical Flat Plate to a Non-Newtonian Fluid, J. Heat Transf., Vol. 111, 1989, pp. 189-191.

[8] D. V. Boger. Demonstration of Upper and Lower Newtonian Fluid Behaviour in a Pseudoplastic Fluid, Nature, Vol. 265, 1977, pp. 126-128.

[9] L. S.Yao, M. M. Molla. Non-Newtonian Fluid Flow on a Flat Plate Part I: Boundary Layer, J. Thermophys. Heat Transf., Vol. 22, 2008, pp.758-761.

[10] M. M. Molla, L. S. Yao. Non-Newtonian Fluid Flow on a Flat Plate Part II: Heat Transfer, J. Thermophys. Heat Transf., Vol. 22, 2008, pp.762-765.

[11] L. S. Yao, M. M. Molla. Forced Convection of NonNewtonian Fluids on a Heated Flat Plate, Int. J. Heat Mass Transf., Vol. 51, 2008, pp.5154-5159.

[12] M. M. Molla, L. S. Yao. The Flow of Non-Newtonian Fluid on a Flat Plate with a Uniform Heat Flux, J. Heat Transf., vol. 131, 2009, 011702-1 6.

[13] M. M. Molla, L. S. Yao. Non-Newtonian Natural Convection Along a Vertical Heated Wavy Surface using a Modified Power-law Viscosity Model, J. Heat Transf., 131, 2009, 012501-1 6.

[14] M. M. Molla, L. S. Yao. Non-Newtonian Natural Convection Along a Vertical Plate Heated with Uniform Surface Heat Fluxes, J. Thermophys. Heat Transf., Vol. 23, 2009, pp.176-185.

[15] M. M. Molla, L. S. Yao. Mixed Convection of NonNewtonian Fluids Along a Heated Vertical Flat Plate, Int. J. Heat Mass Transf., Vol. 52, 2009, pp. 3266-3271. 
[16] S. Ghosh Moulic, L. S. Yao. Non-Newtonian Natural Convection Along a Vertical Flat Plate with Uniform pp. 062501-1 8 . Surface Temperature, J. Heat Transf., Vol. 131, 2009, 